\title{
Data Reconstruction for a Disturbed Soil-Column Experiment Using an Optimal Perturbation Regularization Algorithm
}

\author{
Gongsheng $\mathrm{Li}_{,}{ }^{1} \mathrm{De} \mathrm{Yaor}^{2}$ and Yongzai Wang ${ }^{3}$ \\ ${ }^{1}$ Institute of Applied Mathematics, Shandong University of Technology, Zibo, Shandong 255049, China \\ ${ }^{2}$ Institute of Mining Technology, Inner Mongolia University of Technology, Hohhot, \\ Inner Mongolia 010051, China \\ ${ }^{3}$ Analysis and Testing Center, Shandong University of Technology, Zibo, Shandong 255049, China
}

Correspondence should be addressed to Gongsheng Li, ligs@sdut.edu.cn

Received 9 August 2011; Revised 18 November 2011; Accepted 19 November 2011

Academic Editor: Morteza Rafei

Copyright (C) 2012 Gongsheng Li et al. This is an open access article distributed under the Creative Commons Attribution License, which permits unrestricted use, distribution, and reproduction in any medium, provided the original work is properly cited.

This paper deals with data reconstruction problem for a real disturbed soil-column experiment using an optimal perturbation regularization algorithm. A purpose of doing the experiment is to simulate and study transport behaviors of $\mathrm{Ca}^{2+}, \mathrm{Na}^{+}, \mathrm{Mg}^{2+}, \mathrm{K}^{+}, \mathrm{SO}_{4}{ }^{2-}, \mathrm{NO}_{3}{ }^{-}, \mathrm{HCO}_{3}{ }^{-}$, and $\mathrm{Cl}^{-}$ when they vertically penetrating through sandy soils. By data analysis to breakthrough data of the eight kinds of solute ions, two kinds of models describing their transport behaviors in the column are given. One is the advection-dispersion equation with time-dependent reaction terms suitable for three ions of $\mathrm{HCO}_{3}{ }^{-}, \mathrm{NO}_{3}{ }^{-}$, and $\mathrm{K}^{+}$, the other is the ordinary advection-dispersion equation suitable for the rest ions. Furthermore, all the unknowns in each model are determined by utilizing the optimal perturbation regularization algorithm, respectively, and then the breakthrough data for each considered ion are reconstructed successfully. The inversion results show that the advectiondispersion model with suitable time-dependent reaction terms can be utilized to describe the experimental process and reconstruct the experimental data.

\section{Introduction}

Soil and groundwater pollution has become a serious threat to sustainable development throughout the world. It is important to characterize physical/chemical reactions quantitatively for a solute transport process in the soils and groundwater (see [1-4], e.g.). To understand transport behaviors of the soil in the presence of infiltrating contaminants, soil column experiments are often performed in the laboratory. There are disturbed and undisturbed soil-column infiltrating experiments, and the soil-column in a disturbed experiment is often 
loaded uniformly and orderly with a lucite tube. The primary purpose of doing a disturbed soil-column experiment is to simulate and study transport behavior of the considered ion when it vertically penetrating through the column with artificial chemical liquid.

On the research of soil-column experiments in the known literatures we have, 1D case is often concerned with. In early studies, it is a main task to construct mathematical models for soil-column experiments. The typical works could belong to Nielsen and van Genuchten and their researching groups. Nielsen et al. (see [5], e.g.) gave a general advectiondispersion equation describing solute transport in unsaturated soils, and van Genuchten and Wagenet (see [6], e.g.) discussed solute transport models of two sites/two regions in the soils. With development of computational tools and techniques, numerical methods and software packages based on convection-dispersion equations are widely utilized in the research of soilcolumn infiltrating experiments (see [7-13], e.g.). Recently, the authors have ever considered an undisturbed soil-column experiment using a linear model (see [14]) and a nonlinear model (see [15]), respectively, and gave successful numerical inversions by utilizing the optimal perturbation algorithm based on the measured breakthrough data.

As we know, for a disturbed soil-column infiltrating experiment, there always has no more complicated physical/chemical reactions in the solute transport process, and then a mathematical model seems to be easy to establish due to linearity of the transportation. However, it is still a trouble on how to uniquely determine transport parameters in the model which can not be measured directly by the experiment. Another thing is how to work out the unknown parameters with high precision as much as possible. Thus, inverse problem and inversion analysis methods are often encountered with for solutes transport problems in soilcolumn infiltrating experiments.

In this paper, we will investigate a disturbed soil-column experiment carried out in Zibo, Shandong Province, China, and study transport behaviors of eight kinds of ions of $\mathrm{Ca}^{2+}, \mathrm{Na}^{+}, \mathrm{Mg}^{2+}, \mathrm{K}^{+}, \mathrm{SO}_{4}{ }^{2-}, \mathrm{NO}_{3}{ }^{-}, \mathrm{HCO}_{3}{ }^{-}$, and $\mathrm{Cl}^{-}$by the inversion method. On the basis of advection-dispersion mechanism and hydrochemical analysis to the experimental data, two kinds of models describing their transport behaviors in the column are discussed respectively. By applying the optimal perturbation regularization algorithm, all unknown transport parameters for each ion are determined, and then the measured breakthrough data are reconstructed successfully. The inversion results show that the reconstruction data basically coincide with the measured breakthrough data, and the advection dispersion equation with suitable time-dependent reaction terms can be utilized to describe the experimental process and reconstruct the experimental data. The paper is arranged as follows.

Section 2 gives the soil-column experiment, breakthrough curves of the eight ions and preliminary analysis of their transport behaviors. In Section 3, two kinds of transport models are given respectively, one is suitable for $\mathrm{HCO}_{3}{ }^{-}, \mathrm{NO}_{3}{ }^{-}$, and $\mathrm{K}^{+}$, and the other is for the rest. With the help of the models, the parameter identification problem for each ion is set forth based on the breakthrough data, and an optimal perturbation regularization algorithm is introduced. In Section 4, all the unknowns for each considered ion are determined by the inversion algorithm, and the breakthrough data are reconstructed. Finally, conclusions and discussions on the real problem here are presented.

\section{Experimental Analysis}

The disturbed soil-column experiment was carried out in a simple apparatus in a lab in Shandong University of Technology. The device is installed with three systems which are 

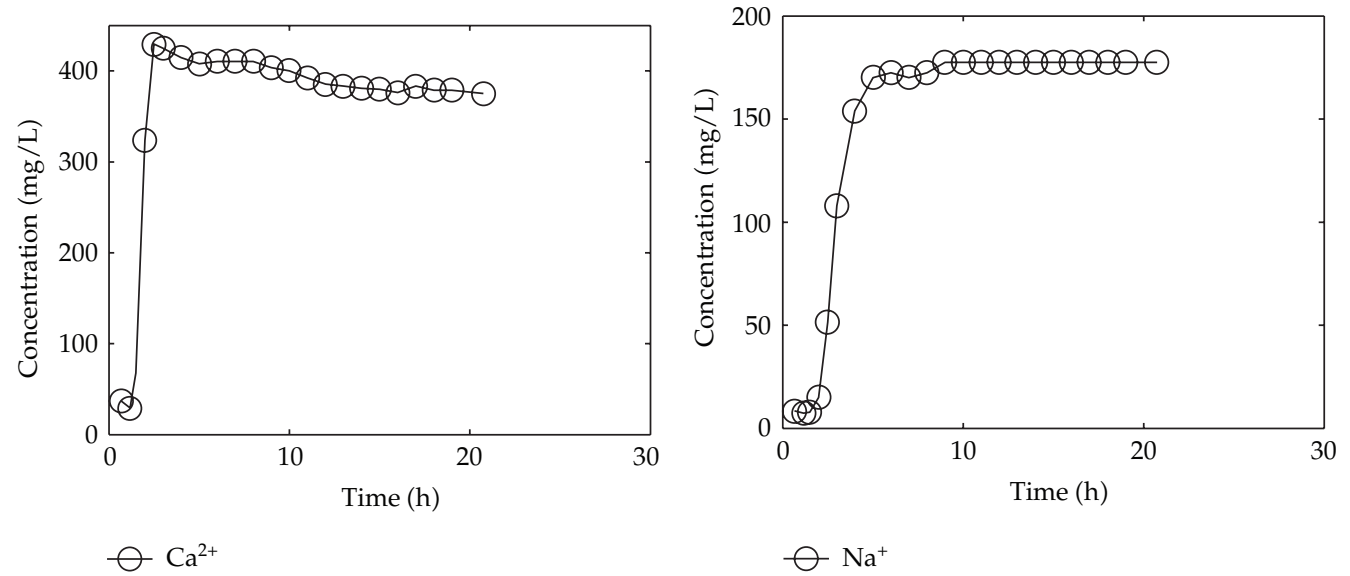

(a)

(b)

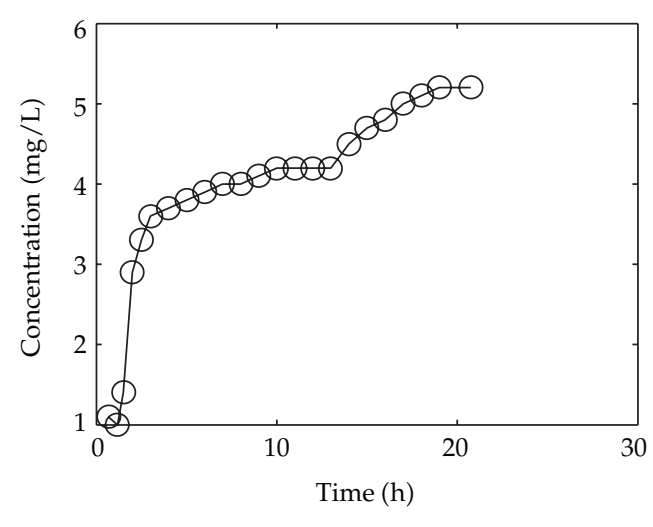

$\odot \mathrm{K}^{+}$

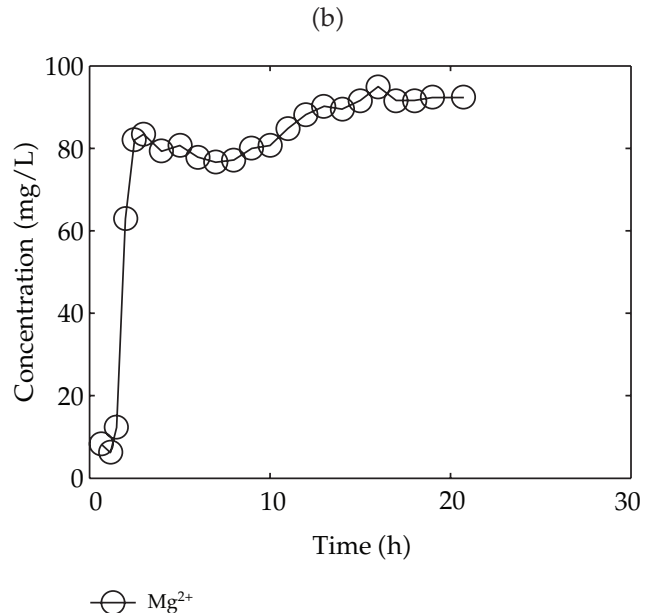

(d)

Figure 1: Breakthrough data of positive solute ions in the experiment.

infiltrating system, soil-column system, and sample collector system, respectively. The soil column is composed by fine sands with diameter of $2[\mathrm{~mm}]$, the diameter of the lucite tube loading the column is $18.6[\mathrm{~cm}]$, and the height of the column is about $62[\mathrm{~cm}]$. After infiltrating the column with distilled water for 24 hours, the experiment was performed by infiltrating into the column with artificial coalmine water at the temperature of 20 centigrade.

By collecting water samples at the bottom of the column, 23 samples were collected, and all of them were immediately sent to tested and analyzed in Shandong General Inspecting Station of Geology and Environment. Thus, we get the so-called breakthrough data of positive ions of $\mathrm{Ca}^{2+}, \mathrm{Na}^{+}, \mathrm{K}^{+}$, and $\mathrm{Mg}^{2+}$ and negative ions of $\mathrm{Cl}^{-}, \mathrm{SO}_{4}{ }^{2-}, \mathrm{HCO}_{3}^{-}$, and $\mathrm{NO}_{3}{ }^{-}$, which are plotted in Figure 1 and Figure 2, respectively, where the transverse axis denotes the experimental time $[\mathrm{h}]$, and the vertical axis represents concentration of the solute ion at the bottom of the column $[\mathrm{mg} / \mathrm{L}]$.

In Figure 1, we find that $\mathrm{Ca}^{2+}, \mathrm{Na}^{+}$, and $\mathrm{Mg}^{2+}$ in the liquid phase almost have same behaviors which go up quickly at the initial stage and then tend to steady situations gradually 

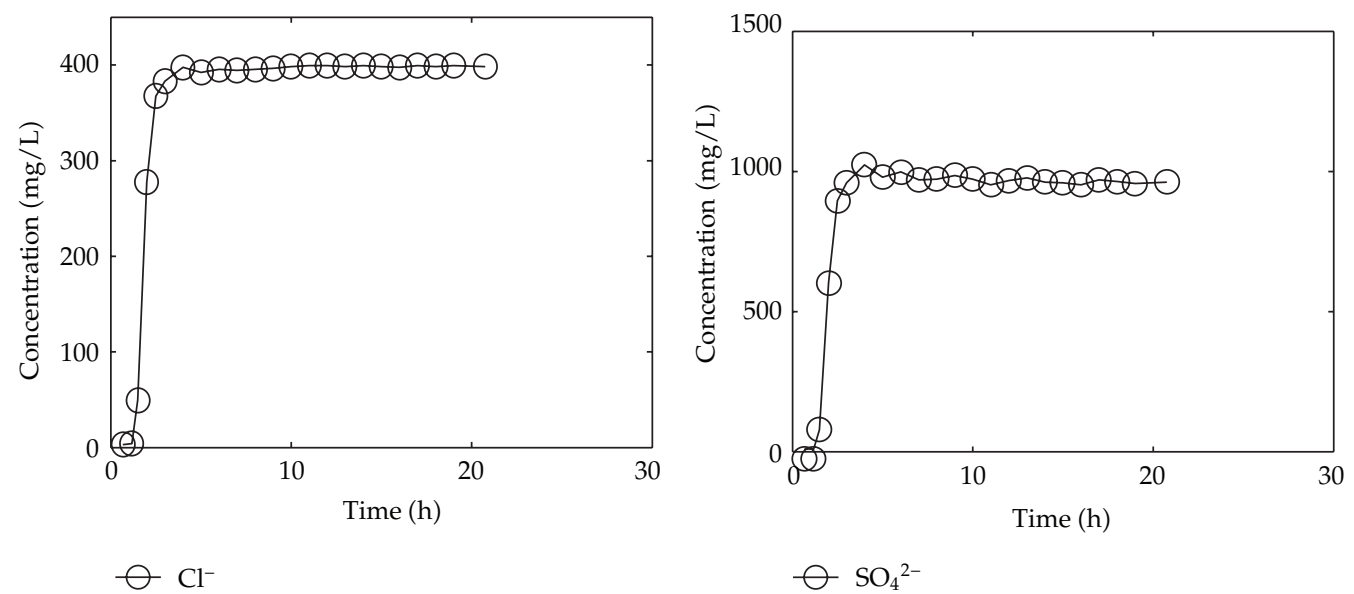

(a)

(b)

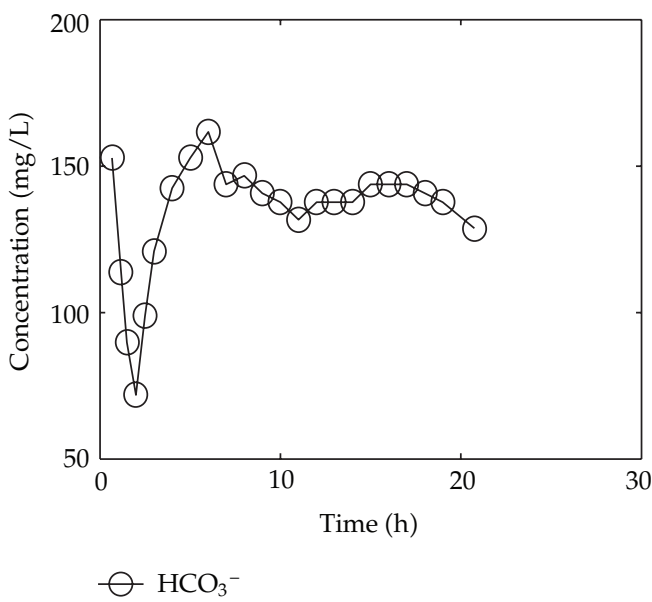

(c)

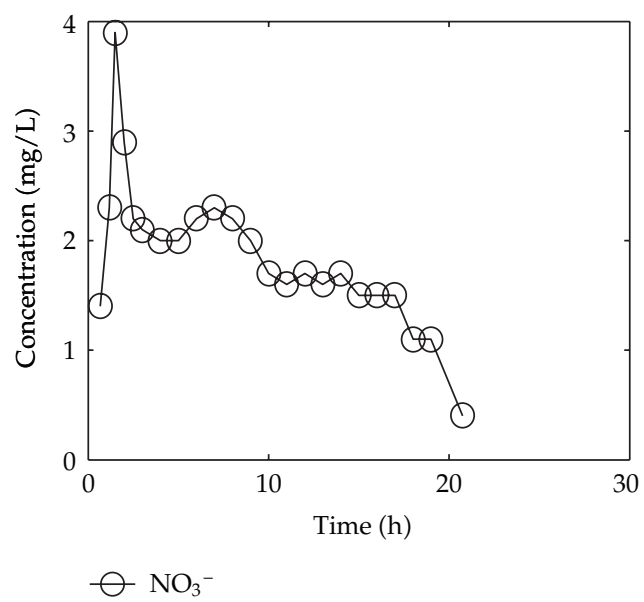

(d)

Figure 2: Breakthrough data of negative solute ions in the experiment.

after 2-3 hours; however, there is a different performance for $\mathrm{K}^{+}$. Although $\mathrm{K}^{+}$goes up rapidly during the initial stage of 3 hours, it is still in an increasing trend at a slow speed after $t=3[\mathrm{~h}]$.

On the other hand, let us investigate behaviors of negative ions plotted in Figure 2. $\mathrm{Cl}^{-}$and $\mathrm{SO}_{4}{ }^{2-}$ have the same tendency with $\mathrm{Ca}^{2+}, \mathrm{Na}^{+}$, and $\mathrm{Mg}^{2+}$, but the situations are complicated for $\mathrm{HCO}_{3}{ }^{-}$and $\mathrm{NO}_{3}{ }^{-}$. It is noticeable that the measured initial concentration of $\mathrm{HCO}_{3}{ }^{-}$in the inflow is very small, and the first sample (at $t=2 / 3[\mathrm{~h}]$ ) gives a high concentration which is $152.7[\mathrm{mg} / \mathrm{L}]$. The reason may be ascribed to the actions of free $\mathrm{CO}_{2}$ in the inflow. From $t=2 / 3$ [h] to $t=2[\mathrm{~h}]$, the concentration goes down rapidly, it goes up quickly from $t=2$ [h] to $t=6[\mathrm{~h}]$, and approaches to an equilibrium after $t=6[\mathrm{~h}]$. As for $\mathrm{NO}_{3}{ }^{-}$, its first breakthrough concentration at $t=2 / 3[\mathrm{~h}]$ is $1.4[\mathrm{mg} / \mathrm{L}]$, which is lower than that of $1.7[\mathrm{mg} / \mathrm{L}]$ in the inflow, and after $t=2 / 3[\mathrm{~h}]$, it goes up rapidly during $t \in(2 / 3,3 / 2)$ $[\mathrm{h}]$, and then goes down rapidly until $t=3[\mathrm{~h}]$. It is surprising that it still goes down after $t=3[\mathrm{~h}]$ but with small oscillations. 
In the following, on the basis of the above analysis, two kinds of mathematical models describing the solutes transport behaviors in the column will be given respectively, and an optimal perturbation algorithm will be introduced to determine the unknown parameters in the models.

\section{Mathematical Model and Inversion Algorithm}

\subsection{The Mathematical Models}

It is wellknown that, under suitable hypotheses, solute transportation in homogeneous porous media can be characterized using 1D advection-dispersion equation. For the disturbed soil-column experiment discussed in Section 2, physical/chemical reactions between the liquid and solid phases can be ignored since the column is composed by clean silver sands, and all of the ions transport in the column could be dominated by hydrologic dispersion and chemical reactions/ions exchanges among the solutes ions in the liquid phase. On the other hand, on the basis of the analysis in Section 2, we can deduce that transport behaviors of the ions of $\mathrm{Ca}^{2+}, \mathrm{Na}^{+}, \mathrm{Mg}^{2+}$, and $\mathrm{Cl}^{-}$with $\mathrm{SO}_{4}{ }^{2-}$ could be described by the ordinary advection-dispersion equation, and transport model for the ions of $\mathrm{HCO}_{3}{ }^{-}, \mathrm{K}^{+}$, and $\mathrm{NO}_{3}{ }^{-}$ could be different, but can be described by the advection-dispersion equation with different time-dependent reaction terms representing possible chemical reactions/ions exchanges depending on the experimental time.

Based on the above analysis, the ions of $\mathrm{Ca}^{2+}, \mathrm{Na}^{+}, \mathrm{Mg}^{2+}$, and $\mathrm{Cl}^{-}$with $\mathrm{SO}_{4}{ }^{2-}$ almost have similar behaviors resulting from convection and dispersion mechanisms. Without loss of generality, taking $\mathrm{SO}_{4}{ }^{2-}$ as example, and denoting $c_{1}=c_{1}(x, t)$ as its concentration in the liquid phase, then there is the ordinary advection-dispersion equation given below:

$$
\frac{\partial c_{1}}{\partial t}=a_{L} v \frac{\partial^{2} c_{1}}{\partial x^{2}}-v \frac{\partial c_{1}}{\partial x}, \quad 0<x<l, t_{0}<t<T_{\text {tol }}
$$

where $a_{L}$ is the longitudinal dispersivity, $v$ is the average flow velocity, $l$ is the length of the column, $t_{0}$ is the time at which the first sample collected, and $T_{\text {tol }}$ is the total infiltrating time.

Next, due to specialties of concentrations of $\mathrm{HCO}_{3}{ }^{-}, \mathrm{K}^{+}$, and $\mathrm{NO}_{3}{ }^{-}$varying with the experimental time, we will utilize time-dependent reaction terms to describe their transport behaviors, respectively. Denote $c_{i}=c_{i}(x, t)(i=2,3,4)$ as their concentrations of $\mathrm{HCO}_{3}{ }^{-}, \mathrm{K}^{+}$ and $\mathrm{NO}_{3}{ }^{-}$, respectively, then we have

$$
\frac{\partial c_{i}}{\partial t}=a_{L} v \frac{\partial^{2} c_{i}}{\partial x^{2}}-v \frac{\partial c_{i}}{\partial x}+\beta_{i-1}(t) c_{i}, \quad 0<x<l, t_{0}<t<T_{\mathrm{tol}}, i=2,3,4,
$$

where $\beta_{i-1}(t)(i=2,3,4)$ represent the physical/chemical reactions depending on the experimental time for solutes ions of $\mathrm{HCO}_{3}{ }^{-}, \mathrm{K}^{+}$, and $\mathrm{NO}_{3}{ }^{-}$, respectively, which are unknown in the experiment.

Finally, following the method as used in paper [15], initial and boundary conditions of (3.1) and (3.2) are given as follows.

$$
c_{i}\left(x, t_{0}\right)=c_{i 0}+\frac{\left(c_{i}\left(l, t_{0}\right)-c_{i 0}\right) x^{m_{i}}}{l^{m_{i}}}, \quad 0 \leq x \leq l, i=1,2,3,4
$$


here $m_{i}>0(i=1,2,3,4)$ are unknown initial indexes which will be determined later. In addition, the boundary conditions are given as:

$$
c_{i}(0, t)=c_{i 0}, \quad \frac{\partial c_{i}}{\partial x}(l, t)=0, \quad t_{0} \leq t \leq T_{\mathrm{tol}}, i=1,2,3,4
$$

Then, two kinds of mathematical models describing solutes transport behaviors in the soil-column are given by (3.1) and (3.2) together with the initial boundary value conditions (3.3) and (3.4), respectively. Since the initial indexes in condition (3.3) and the reaction coefficient functions in (3.2) are all unknown, a suitable inversion algorithm must be applied to determine them numerically. Furthermore, thanks to the independence of (3.1) with (3.2), we can see in the next section that each ion can be reconstructed independently of the others.

In the following, we will take $\mathrm{HCO}_{3}{ }^{-}\left(c_{2}\right)$ as example and introduce an optimal perturbation regularization algorithm (see [14-16]), e.g.) to determine the unknown parameters based on the measured breakthrough data. For convenience of computation, we will transform the model to a dimensionless form.

Set $C_{2}=c_{2} / c_{20}, Z=x / l$ and $T=v t / l$, then we have

$$
\frac{\partial C_{2}}{\partial T}=\frac{a_{L}}{l} \frac{\partial^{2} C_{2}}{\partial Z^{2}}-\frac{\partial C_{2}}{\partial Z}+\frac{l}{v} q_{1}(T) C_{2}, \quad 0<Z<1, T_{0}<T<\bar{T},
$$

where $q_{1}(T)=\beta_{1}((l / v) T)$, and $T_{0}=v t_{0} / l, \bar{T}=v T_{\mathrm{tol}} / l$.

The initial and boundary value conditions are transformed to

$$
\begin{gathered}
C_{2}\left(Z, T_{0}\right)=1+\left(\frac{c_{2}\left(l, t_{0}\right)}{c_{20}}-1\right) Z^{m_{2}}, \quad 0 \leq Z \leq 1, \\
C_{2}(0, T)=1, \quad \frac{\partial C_{2}}{\partial Z}(1, T)=0, \quad T_{0} \leq T \leq \bar{T},
\end{gathered}
$$

respectively.

Now the problem left is to determine the initial parameter $m_{2}$ and the reaction coefficient $q_{1}(T)$ which can lead to an inverse problem of parameter identification. Therefore, some additional information from the experiment is needed. The additional condition we will utilize is the breakthrough data as plotted in Figure 2. Also by dimensionless to the real data, we have

$$
C_{2}\left(1, T_{k}\right)=C_{2}^{k}, \quad k=1,2, \ldots, K
$$

As a result for $\mathrm{HCO}_{3}{ }^{-}$, an inverse problem of determining the time-dependent reaction function and the initial index is formulated by (3.5), the initial boundary condition (3.6)-(3.7), and the over-posed condition (3.8).

In addition, the initial concentration of $\mathrm{SO}_{4}{ }^{2-}\left(c_{1}\right), \mathrm{HCO}_{3}{ }^{-}\left(c_{2}\right), \mathrm{K}^{+}\left(c_{3}\right)$, and $\mathrm{NO}_{3}{ }^{-}\left(c_{4}\right)$ are given as $c_{10}=1023.2[\mathrm{mg} / \mathrm{L}], c_{20}=34.1[\mathrm{mg} / \mathrm{L}], c_{30}=17.2[\mathrm{mg} / \mathrm{L}]$, and $c_{40}=1.7$ $[\mathrm{mg} / \mathrm{L}]$, respectively. By the experiment, we know that the length of the column is $l=0.62$ [m], the average flow velocity is $v=2.4 \times 10^{-5}[\mathrm{~m} / \mathrm{s}]$, and the total infiltrating time is $T_{\text {tol }}=21[\mathrm{~h}]$. Finally, noting that the soil medium is composed by sand with $2[\mathrm{~mm}]$ diameter, 
the longitudinal dispersivity $a_{L}=8.8 \times 10^{-3}[\mathrm{~m}]$, which is estimated by known experimental results (see [1], e.g.).

\subsection{The Optimal Perturbation Regularization Algorithm}

In this subsection, we will also take $\mathrm{HCO}_{3}{ }^{-}\left(c_{2}\right)$ as example to introduce the optimal perturbation regularization algorithm. Suppose that the unknown source parameter has an approximate polynomial expansion given as:

$$
q_{1}(T) \approx \sum_{i=0}^{N} a_{i} T^{i}
$$

where $a_{i}(i=0,1, \ldots, N)$ are expansion coefficients. Then, the model parameters we are to determine can be denoted by a vector given as follows:

$$
r=\left(a_{0}, a_{1}, \ldots, a_{N}, m_{2}\right),
$$

here $a_{i}(i=0,1, \ldots, N)$ are coefficients referred to (3.9), and $m_{2}$ is the initial index given in (3.6).

Now, for any prescribed $r_{j}(j=0,1, \ldots)$, set

$$
r_{j+1}=r_{j}+\delta r_{j}, \quad j=0,1, \ldots
$$

Thus in order to get $r_{j+1}$ from given $r_{j}$, we need to compute an optimal increasing perturbation $\delta r_{j}$. In the following, for convenience of writing, $r_{j}$ and $\delta r_{j}$ are abbreviated as $r$ and $\delta r$, respectively.

Denote $C_{2}(Z, T ; r)$ as the unique solution of the forward problem (3.5)-(3.7) for any prescribed vector $r$. Taking Taylor's expansion for $C_{2}(1, T ; r+\delta r)$ at $r$ and ignoring higher order terms, we can get

$$
C_{2}(1, T ; r+\delta r) \approx C_{2}(1, T ; r)+\nabla^{T} C_{2}(1, T ; r) \cdot \delta r .
$$

Furthermore, define a perturbation functional for $\delta r$ as follows:

$$
F(\delta r)=\left\|C_{2}\left(1, T_{k} ; r\right)+\nabla^{T} C_{2}\left(1, T_{k} ; r\right) \cdot \delta r-C_{2}^{k}\right\|^{2}+\alpha\|\delta r\|^{2},
$$

where $\alpha>0$ is regularization parameter, and

$$
\nabla^{T} C_{2}\left(1, T_{k} ; r\right) \cdot \delta r \approx \sum_{i=0}^{N+1} \frac{C_{2}\left(1, T_{k} ; r+\tau_{i}\right)-C_{2}\left(1, T_{k} ; r\right)}{\tau_{i}} \delta r_{i}, \quad k=1,2, \ldots, K
$$

$\tau=\left(\tau_{0}, \tau_{1}, \ldots, \tau_{N+1}\right)$ is numerical differential steps vector. 
Denote

$$
\begin{array}{cc}
\xi=\left(C_{2}\left(1, T_{1} ; r\right), C_{2}\left(1, T_{2} ; r\right), \ldots, C_{2}\left(1, T_{K} ; r\right)\right) ; & \eta=\left(C_{2}^{1}, C_{2}^{2}, \ldots, C_{2}^{K}\right), \\
g_{k i}=\frac{C_{2}\left(1, T_{k} ; r+\tau_{i}\right)-C_{2}\left(1, T_{k} ; r\right)}{\tau_{i}}, \quad G=\left(g_{k i}\right)_{K \times(N+2)} .
\end{array}
$$

Then, the functional expression (3.13) can be reduced to

$$
F(\delta r)=\|G \delta r-(\eta-\xi)\|^{2}+\alpha\|\delta r\|^{2} .
$$

It is easy to testify that minimizing (3.17) can be reduced to solve the following normal equation (see [17], e.g.)

$$
G^{T} G \delta r+\alpha \delta r=G^{T}(\eta-\xi)
$$

By the above discussions, we know that the best perturbation $\delta r$ can be solved by the normal equation (3.18) via

$$
\delta r=\left(\alpha I+G^{T} G\right)^{-1} G^{T}(\eta-\xi)
$$

then the optimal coefficient vector $r$ can be approximated by iterations (3.11) as long as the perturbation satisfying a given precision. This is the principal idea and procedure of the optimal perturbation regularization algorithm. The key points of performing the algorithm lie in appropriate choices of initial iteration $r_{0}$, regularization parameter $\alpha$, and differential steps vector $\tau=\left(\tau_{0}, \tau_{1}, \ldots, \tau_{N+1}\right)$.

\section{Inversion Results}

Following the methods as used in paper [14, 15], we will implement the above optimal perturbation regularization algorithm to determine the unknown vector $r$ given by (3.10) numerically, and then reconstruct the measured breakthrough data for the ions of $\mathrm{HCO}_{3}{ }^{-}$, $\mathrm{SO}_{4}{ }^{2-}, \mathrm{K}^{+}$, and $\mathrm{NO}_{3}{ }^{-}$, respectively. The reaction coefficient can be determined in different approximate space, and one needs to choose a suitable approximate dimension $N$ so as to reconstruct the measured data with high accuracy. For the four kinds of ions, we will realize the inversion with different dimension. All computations are performed in a PC of Dell Dimension 9200.

\subsection{Data Reconstruction for $\mathrm{HCO}_{3}{ }^{-}$}

Set $N=7$ and choose regularization parameter as $\alpha=0.08$, initial iteration as $r_{0}=$ $(0,0,0,0,0,0,1)$, and numerical differential steps vector as $\tau=(1 e-1,1 e-2, \ldots, 1 e-9)$, then 


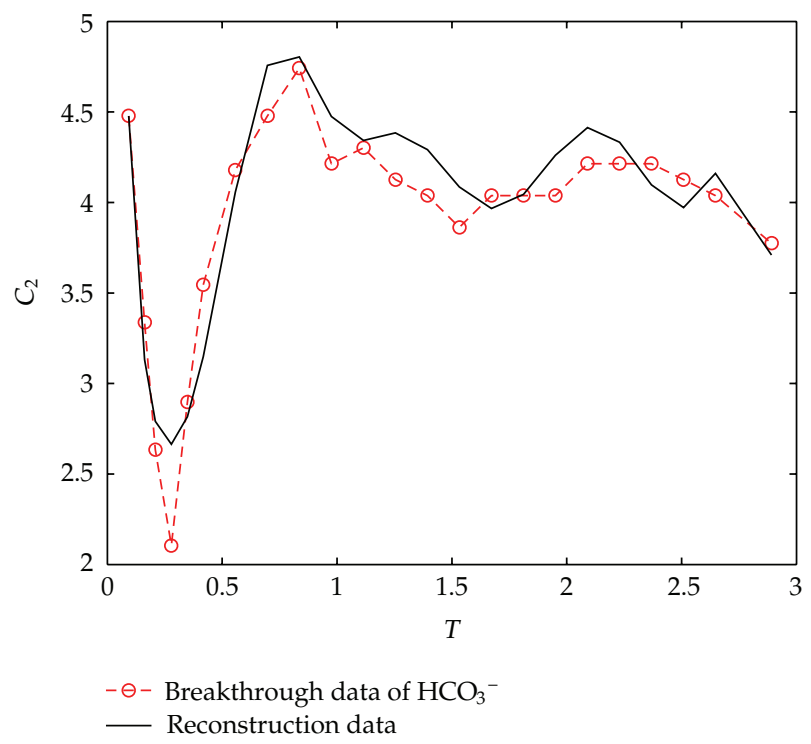

Figure 3: The breakthrough data and reconstruction data for $\mathrm{HCO}_{3}{ }^{-}$.

by 100 iterations which cost CPU time 295.4 [s], we obtain the inversion parameters given as follows:

$$
r^{\text {inv }}=(-1.7124,11.1543,-18.0604,5.9008,9.3842,-9.3487,3.1368,-0.3681,1.0727) ，
$$

that is,

$$
\begin{aligned}
q_{1}(T)= & -1.7124+11.1543 T-18.0604 T^{2}+5.9008 T^{3}+9.3842 T^{4} \\
& -9.3487 T^{5}+3.1368 T^{6}-0.3681 T^{7} \\
m_{2}= & 1.0727
\end{aligned}
$$

and the relative inversion error is worked out as

$$
\text { Err }=\left\|\eta-C_{2}\left(1, T ; r^{\text {inv }}\right)\right\|_{2} /\|\eta\|_{2}=0.0113
$$

where $\eta$ is the measured breakthrough data vector of $\mathrm{HCO}_{3}{ }^{-}$given in (3.15), and $C_{2}\left(1, T ; r^{\text {inv }}\right)$ is the reconstruction data. Furthermore, the inversion reconstruction data and the real measured breakthrough data are plotted in Figure 3.

\subsection{Data Reconstruction for $\mathrm{SO}_{4}{ }^{2-}$}

Let us consider the solute ion of $\mathrm{SO}_{4}{ }^{2-}$. As for (3.1) with corresponding initial boundary conditions, there is only one initial parameter $m_{1}$ to be determined. Therefore, the optimal perturbation algorithm can be simplified as done in paper [14]. By utilizing the optimal 


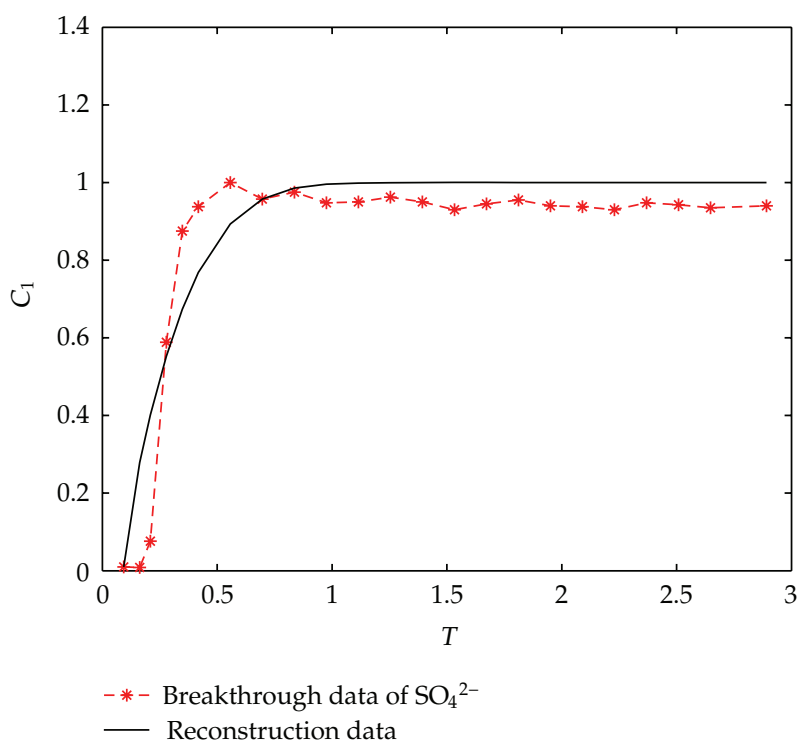

Figure 4: The breakthrough data and reconstruction data for $\mathrm{SO}_{4}{ }^{2-}$.

perturbation algorithm without regularization term (choosing regularization parameter $\alpha=$ 0 ), and taking initial iteration value as 1 , numerical differential step as $\tau=1 e-1$, the initial index can be determined by 7 iterations costing CPU time 1.7 [s] which is $m_{1}=3.7588$, and the relative inversion error is Err $=0.0277$. Figure 4 plots the measured breakthrough data and the reconstructed data of $\mathrm{SO}_{4}{ }^{2-}$, respectively.

\subsection{Data Reconstruction for $\mathrm{K}^{+}$and $\mathrm{NO}_{3}{ }^{-}$}

As for $\mathrm{K}^{+}$, similarly as done for $\mathrm{HCO}_{3}{ }^{-}$, take $\mathrm{N}=3$ here and choose regularization parameter as $\alpha=0.05$, initial iteration as $r_{0}=(0,0,0,0,1)$, and numerical differential steps vector as $\tau=(1 e-2,1 e-3,1 e-4,1 e-5,1 e-6)$, the unknown reaction coefficient, and the initial index for $\mathrm{K}^{+}$are worked out by 31 iterations which costing CPU time 24.0 [s] given as follows:

$$
\begin{gathered}
q_{2}(T)=-0.1413-0.1859 T+0.1464 T^{2}-0.0305 T^{3}, \\
m_{3}=0.6558,
\end{gathered}
$$

and the relative inversion error is Err $=0.0141$. Figure 5 plots the measured breakthrough data and the reconstructed data of $\mathrm{K}^{+}$, respectively.

Now let us deal with data reconstruction for $\mathrm{NO}_{3}{ }^{-}$in which case the situation seems to be a little complicated. If still following the above method, there are no inversion results we need to appear. So, we perform the algorithm in a little different way as compared with the above computations by choosing a fixed initial index prescribed. Moreover, if setting an initial index in advance, then we only need to determine the time-dependent reaction coefficient. 


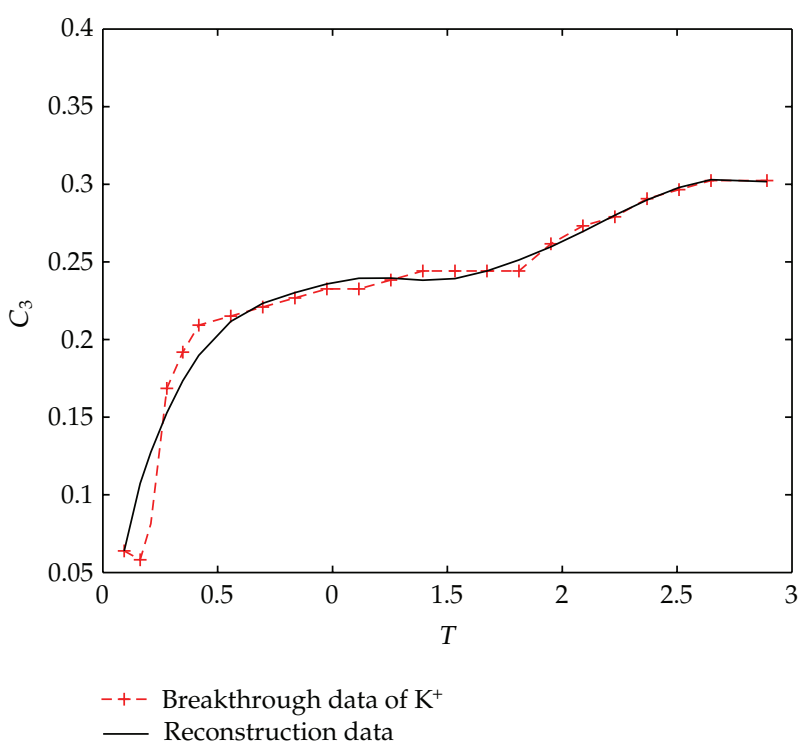

Figure 5: The breakthrough data and reconstruction data for $\mathrm{K}^{+}$.

On the concrete computations, taking $m_{4}=3, N=9$, regularization parameter as $\alpha=0.05$, initial iteration as zero, and numerical differential steps vector as $\tau=(1 e-1,1 e-$ $2, \ldots, 1 e-10)$, an optimal reaction coefficient for $\mathrm{NO}_{3}{ }^{-}$is worked out by 500 iterations which costing CPU time 1636.7 [s] given as follows:

$$
\begin{aligned}
q_{3}(T)= & 3.003-20.104 T+36.064 T^{2}-10.092 T^{3}-21.755 T^{4} \\
& +6.761 T^{5}+17.050 T^{6}-14.783 T^{7}+4.523 T^{8}-0.491 T^{9},
\end{aligned}
$$

and the relative inversion error is Err $=0.0285$. The measured breakthrough data and the reconstruction data for $\mathrm{NO}_{3}{ }^{-}$are plotted in Figure 6, respectively.

\section{Discussions and Conclusions}

\subsection{Discussions}

\subsubsection{On Reaction Coefficient}

Firstly, let us investigate actions of the reaction coefficients. By the inversion result (4.2) for $\mathrm{HCO}_{3}{ }^{-}$, and noting (3.2) (i=2) and its dimensionless form (3.5), we can get

$$
\begin{aligned}
\beta_{1}(t)= & -1.7124+1.5544 t-0.3507 t^{2}+0.0160 t^{3}+0.00354 t^{4} \\
& -4.91 E-4 t^{5}+2.30 E-5 t^{6}-3.76 E-7 t^{7}
\end{aligned}
$$

which is the reaction coefficient with dimensional time $t$ for $\mathrm{HCO}_{3}{ }^{-}$. By (5.1) we can see that the expansion coefficients of $\beta_{1}(t)$ become smaller and smaller as $N$ goes to large. 


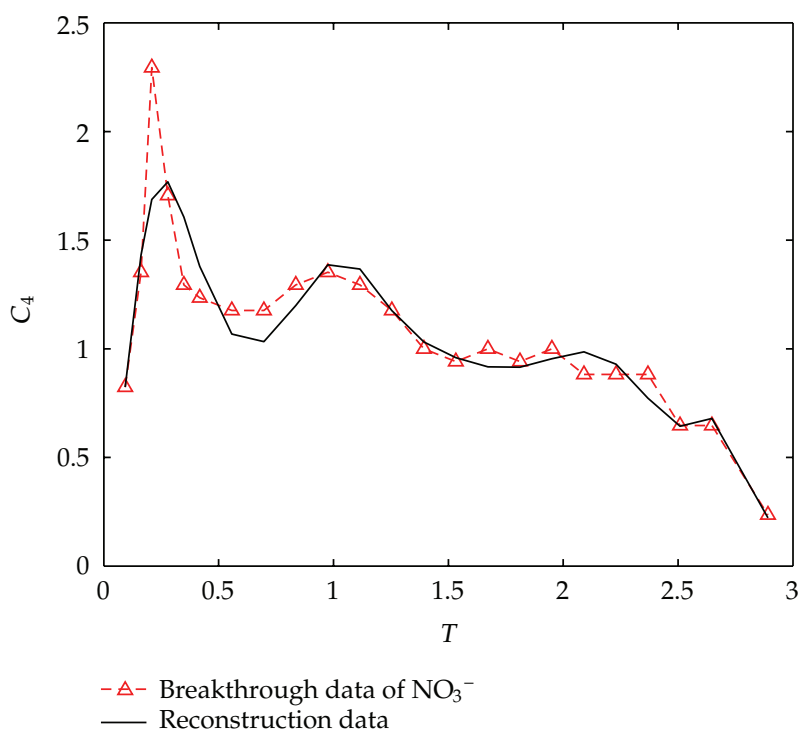

Figure 6: The breakthrough data and reconstruction data for $\mathrm{NO}_{3}{ }^{-}$.

Similarly, the reaction coefficients $\beta_{2}(t)$ and $\beta_{3}(t)$ in $(3.2)(i=2,3)$ can be worked out which are given as

$$
\begin{aligned}
\beta_{2}(t)= & -0.1413-0.0259 t+0.00284 t^{2}-8.26 E-5 t^{3}, \\
\beta_{3}(t)= & 3.003-2.8016 t+0.7004 t^{2}-0.0273 t^{3}-0.00820 t^{4}+3.55 E-4 t^{5} \\
& +1.25 E-4 t^{6}-1.51 E-5 t^{7}+6.43 E-7 t^{8}-9.73 E-9 t^{9}
\end{aligned}
$$

respectively. Furthermore, $\beta_{i}(t)(i=1,2,3)$ are plotted in Figure 7 so as to study their changes with the experimental time, respectively.

From Figure 7, we can find that the reaction to $\mathrm{K}^{+}$almost has no change throughout the experiment; and the reaction to $\mathrm{HCO}_{3}{ }^{-}$goes up at the initial stage, and then keeps a little increase during $t \in(5,15)[\mathrm{h}]$. As for the reaction to $\mathrm{NO}_{3}{ }^{-}$, it decreases during the initial stage, and then keeps unchanging during $t \in(2,15)[\mathrm{h}]$, and after $t>15[\mathrm{~h}]$, it becomes decreasing quickly.

It is noticeable that the above arguments basically coincide with those hydrochemical analysis given in Section 2 except for $\mathrm{NO}_{3}{ }^{-}$in the case of $t>15$ [h]. In addition, we also find that $\beta_{2}(t)$ and $\beta_{3}(t)$ both have similar asymptotic properties as $\beta_{1}(t)$, that is, the expansion coefficients of the reaction term go to zero as $N$ becomes large.

\subsubsection{On Space Distribution at the First Outflow}

Now, look at the following figure which plots space distributions at the first outflow time of $T=T_{0}$ for the four kinds of solute ions.

From Figure 8, we can see that at the first outflow time of $t=2 / 3[\mathrm{~h}], \mathrm{NO}_{3}{ }^{-}, \mathrm{SO}_{4}{ }^{2-}$, and $\mathrm{K}^{+}$are in similar situations, and $\mathrm{HCO}_{3}{ }^{-}$has a special behavior. 


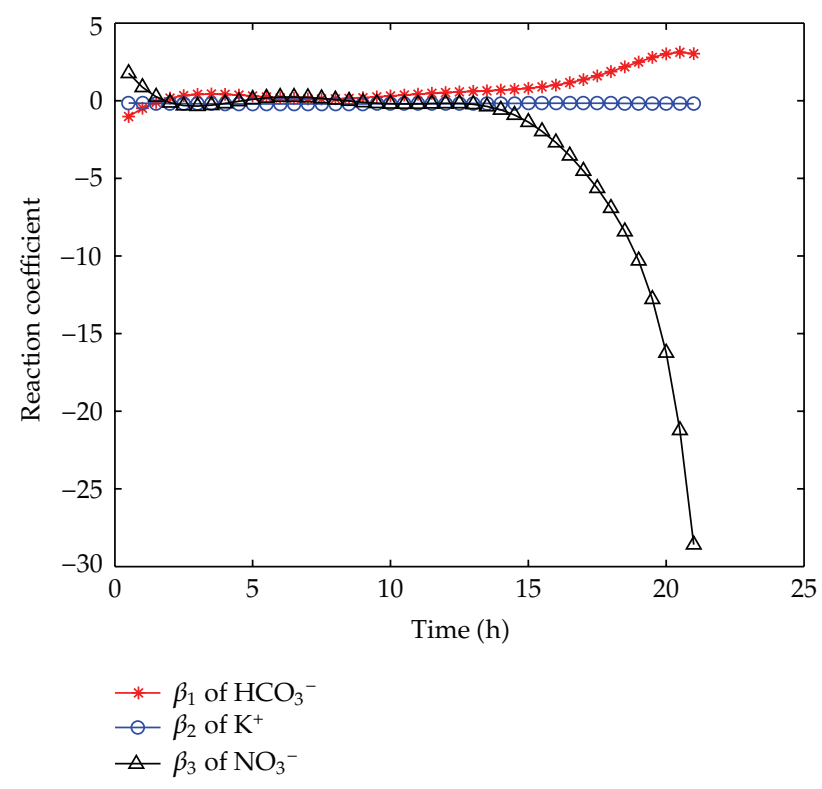

Figure 7: Reaction coefficients with time.

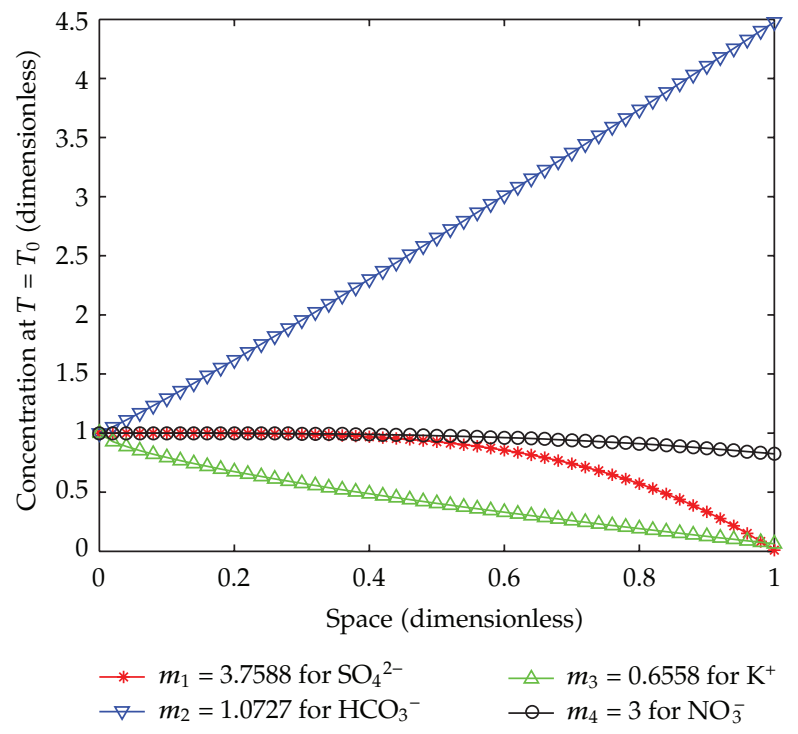

Figure 8: Space distributions at $T=T_{0}$.

For $\mathrm{NO}_{3}{ }^{-}$, its concentration varies from $1(t=0)$ to $1.4 / 1.7 \approx 0.8235(t=2 / 3[\mathrm{~h}])$, and goes down almost along with a straight line very slowly.

For $\mathrm{SO}_{4}{ }^{2-}$, its concentration varies from $1(t=0)$ to $10.0 / 1023.2 \approx 0.0098(t=2 / 3[\mathrm{~h}])$, and goes down with convexity but faster than $\mathrm{NO}_{3}{ }^{-}$.

For $\mathrm{K}^{+}$, its concentration varies from $1(t=0)$ to $1.1 / 17.2 \approx 0.0640(t=2 / 3[\mathrm{~h}])$, and decreases also faster than $\mathrm{NO}_{3}{ }^{-}$, but with concavity. 
As for $\mathrm{HCO}_{3}{ }^{-}$, its concentration changes from $1(t=0)$ to $152.7 / 34.1 \approx 4.4780(t=2 / 3$ $[\mathrm{h}])$, and goes up quickly with almost linearity.

In summary, during this interval of $t \in(0,2 / 3)[\mathrm{h}]$, hydrodynamical advection dispersion actions may be play dominating roles in the solutes transport through the column. However, there are some chemical reactions occurring in $\mathrm{HCO}_{3}{ }^{-}$except for mechanical advection and dispersion. For example, the free $\mathrm{CO}_{2}$ in the inflow can produce $\mathrm{HCO}_{3}{ }^{-}$when penetrating through the column, and the amount of $\mathrm{HCO}_{3}{ }^{-}$reaches a maximum at the bottom of the column at $t=2 / 3[\mathrm{~h}]$.

\subsection{Conclusions}

We give several concluding remarks to end this paper.

(1) By the model and data reconstruction results, we can see that the main transport behaviors of solutes ions in the soil-column are dominated by hydrodynamical advection and dispersion mechanisms. There are no complicated physical/chemical reactions between solid and liquid phases, especially for the solutes ions of $\mathrm{SO}_{4}{ }^{2-}, \mathrm{Cl}^{-}, \mathrm{Ca}^{2+}, \mathrm{Na}^{+}$, and $\mathrm{Mg}^{2+}$, whose transport behaviors through the column can be described by purely advection-dispersion equation (3.1) with suitable initial conditions.

(2) As for $\mathrm{HCO}_{3}{ }^{-}, \mathrm{K}^{+}$, and $\mathrm{NO}_{3}{ }^{-}$, their transport behaviors in the column could be described by the advection-dispersion equation (3.2) with suitable time-dependent reaction terms and initial conditions. It is noticeable that $\mathrm{K}^{+}$has some properties similar to those five ions; but for $\mathrm{HCO}_{3}{ }^{-}$and $\mathrm{NO}_{3}{ }^{-}$, they have some different properties as compared with the other ions maybe resulting from chemical reactions or ions exchanges in the liquid phase.

(3) As stated in Section 5.1.2, at the initial interval of $t \in(0,2 / 3)$ [h], it is still a trouble to describe the solute transport behaviors in mathematics due to lack of measured data. Maybe better experimental devices, and other mathematical tools are needed. In addition, noting that nonlinear processes could occur quickly during this short time, perhaps it is feasible and prospective that utilizing the model in a smaller time scale of second instead of hour during this interval.

(4) By performing the inversion algorithm, we find that a numerical uniqueness on the inverse problem can be obtained in the sense of ignoring higher-order terms. Let us take the inversion for $\beta_{2}(t)$ as example. For the reason of seeking for regularity, we perform the algorithm utilizing the initial index of $m_{3}=0.6558$ determined in Section 4.3, and keeping the same inversion error. Table 1 gives the inversion results for $\beta_{2}(t)$ taking on higher-order polynomials including the case of $N=3$.

From the table, we can find that if taking the first term as an approximation, the reaction coefficient should be $\beta_{2}(t)=-0.14$; if taking the front two terms as an approximation, the optimal reaction coefficient should be given by $\beta_{2}(t)=-0.14-0.02 t$. Another thing we should mention is that for different truncated levels $N=3,4,5,6$ and $N=7$, all of the inversion results preserve the same relative error expressed by Err $=0.0141$. From this point of view, the numerical inversion is of uniqueness too. As to theoretical uniqueness of the inverse problem here, we will deal with it in our sequent papers. 
Table 1: Inversion results for $\beta_{2}(t)$ in higher-order polynomials.

\begin{tabular}{ll}
\hline$N$ & $\beta_{2}(t)$ \\
\hline 3 & $(-0.1410,-0.0260,2.9 e-3,-8.3 e-5)$ \\
4 & $(-0.1400,-0.0265,2.9 e-3,-8.6 e-5,4.2 e-8)$ \\
5 & $(-0.1446,-0.0191,1.3 e-4,3.1 e-4,-2.3 e-5,4.9 e-7)$ \\
6 & $(-0.1409,-0.0222,8.3 e-4,2.7 e-4,-2.6 e-5,8.3 e-7,-8.6 e-9)$ \\
7 & $(-0.1412,-0.0201,7.7 e-6,2.6 e-4,8.3 e-6,-3.9 e-6,2.4 e-7$, \\
\hline
\end{tabular}

\section{Acknowledgment}

This paper is supported by the National Natural Science Foundation of China (Grant nos. 10926194, and 11071148).

\section{References}

[1] N.-Z. Sun, Mathematical Model of Groundwater Pollution, Springer, New York, NY, USA, 1996.

[2] J. Atmadja and A. C. Bagtzoglou, "State of the art report on mathematical methods for groundwater pollution source identification," Environmental Forensics, vol. 2, no. 3, pp. 205-214, 2001.

[3] C. M. Zheng and G. D. Bennett, Applied Contaminant Transport Modeling, John Wiley \& Sons, New York, NY, USA, 2nd edition, 2002.

[4] J. M. Köhne, B. P. Mohanty, and J. Šimůnek, “Inverse dual-permeability modeling of preferential water flow in a soil column and implications for field-scale solute transport," Vadose Zone Journal, vol. 5, no. 1, pp. 59-76, 2006.

[5] D. R. Nielsen, M. Th. van Genuchten, and J. W. Biggar, "Water flow and solute transport process in the unsaturated zone," Water Resources Research, vol. 22, no. 9, pp. 89S-108S, 1986.

[6] M. Th. van Genuchten and R. J. Wagenet, "Two-site/two-region models for pesticide transport and degradation: theoretical development and analytical solutions," Soil Science Society of America Journal, vol. 53, no. 5, pp. 1303-1310, 1989.

[7] N. Toride, F. J. Leij, and M. Th. van Genuchten, “The CXTFIT code for estimating transport parameters from laboratory or field tracer experiments, Version 2.0," type 137, U.S. Department of Agriculture, 1995.

[8] Z. Torsten, "Capability of convection-dispersion transport models to predict transient water and solute movement in undisturbed soil columns," Journal of Contaminant Hydrology, vol. 30, no. 1-2, pp. 101-128, 1998.

[9] L. P. Pang and M. E. Close, "Non-equilibrium transport of Cd in alluvial gravels," Journal of Contaminant Hydrology, vol. 36, no. 1-2, pp. 185-206, 1999.

[10] M. Inoue, J. Šimůnek, S. Shiozawa, and J. W. Hopmans, "Simultaneous estimation of soil hydraulic and solute transport parameters from transient infiltration experiments," Advances in Water Resources, vol. 23, no. 7, pp. 677-688, 2000.

[11] S. K. Kamra, B. Lennartz, M. Th. van Genuchten, and P. Widmoser, "Evaluating non-equilibrium solute transport in small soil columns," Journal of Contaminant Hydrology, vol. 48, no. 3-4, pp. 189-212, 2001.

[12] K. Cui, B. Y. Li, X. S. Li, and G. W. Yang, "Model parameter inversion for cadmium ion transport through unsaturated soils," Advances in Water Science, vol. 15, no. 6, pp. 700-705, 2004 (Chinese).

[13] D. A. Barry, "Effect of nonuniform boundary conditions on steady flow in saturated homogeneous cylindrical soil columns," Advances in Water Resources, vol. 32, no. 4, pp. 522-531, 2009.

[14] G. S. Li, J. Cheng, D. Yao, H. L. Liu, and J. J. Liu, “One-dimensional equilibrium model and source parameter determination for soil-column experiment," Applied Mathematics and Computation, vol. 190, no. 2, pp. 1365-1374, 2007.

[15] G. S. Li, Y. J. Tan, D. Yao, X. Q. Wang, and H. L. Liu, "A non-linear mathematical model for an undisturbed soil-column experiment and source parameter identification," Inverse Problems in Science and Engineering, vol. 16, no. 7, pp. 885-901, 2008. 
[16] C. W. Su, Numerical Methods and Applications of Inverse Problems in PDE (in Chinese), Northwestern Polytechnical University Press, Xi'an, China, 1995.

[17] A. Kirsch, An Introduction to Mathematical Theory of Inverse Problems, vol. 120, Springer, New York, NY, USA, 1996. 


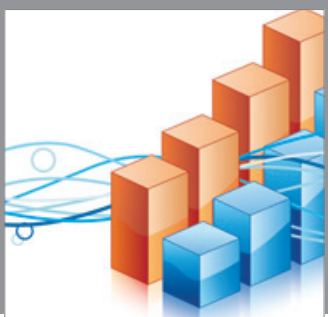

Advances in

Operations Research

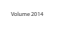

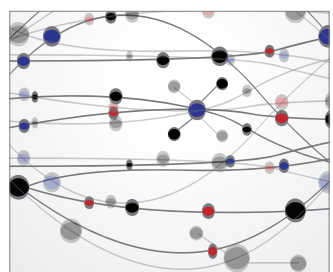

\section{The Scientific} World Journal
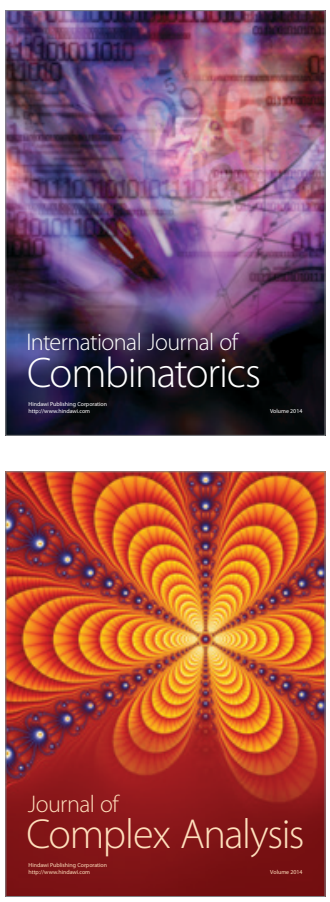

International Journal of

Mathematics and

Mathematical

Sciences
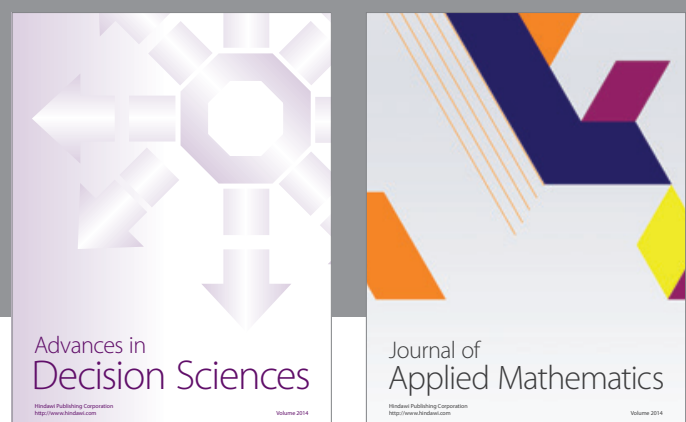

Journal of

Applied Mathematics
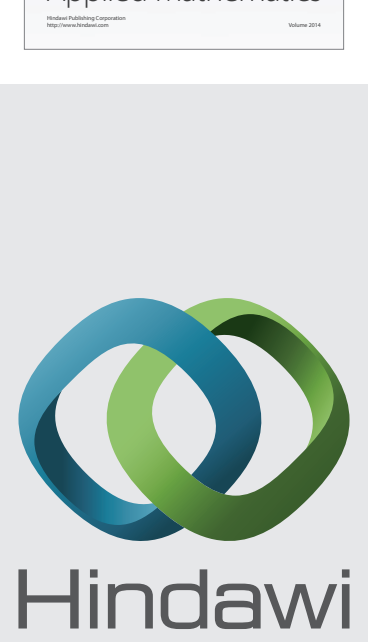

Submit your manuscripts at http://www.hindawi.com
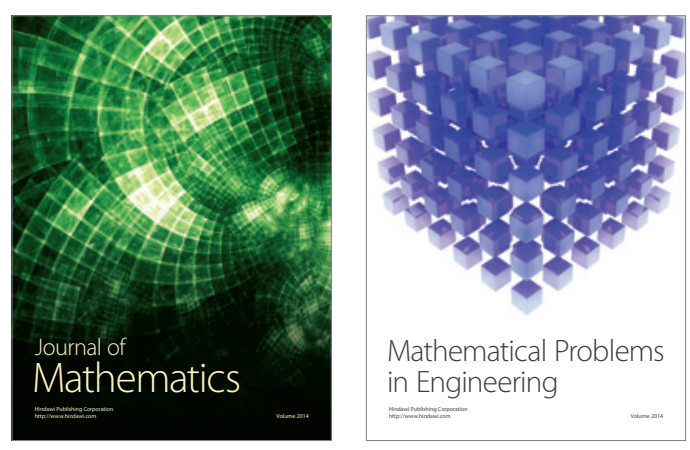

Mathematical Problems in Engineering
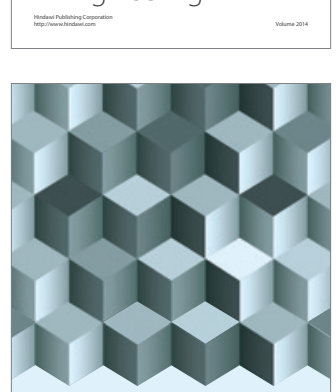

Journal of

Function Spaces
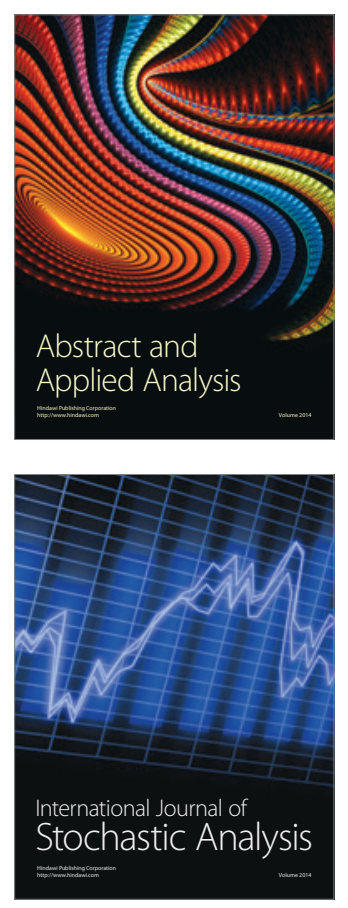

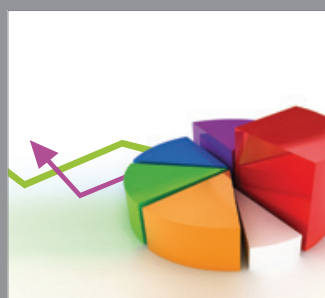

ournal of

Probability and Statistics

Promensencen
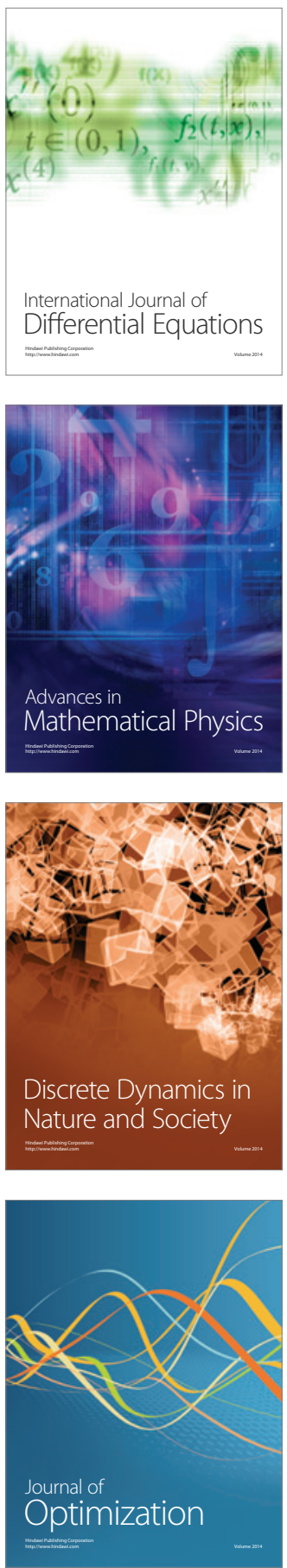